\title{
$(\Phi, \Psi)$-Weak Contractions in Neutrosophic Cone Metric Spaces via Fixed Point Theorems
}

\author{
Wadei F. Al-Omeri $\left(\mathbb{D},{ }^{1}\right.$ Saeid Jafari, ${ }^{2}$ and Florentin Smarandache ${ }^{3}$ \\ ${ }^{1}$ Department of Mathematics, Al-Balqa Applied University, Salt 19117, Jordan \\ ${ }^{2}$ College of Vestsjaelland South, Herrestraede 11, 4200 Slagelse, Denmark \\ ${ }^{3}$ Department of Mathematics, University of New Mexico Gallup, Gallup, NM, USA \\ Correspondence should be addressed to Wadei F. Al-Omeri; wadeimoon1@hotmail.com
}

Received 21 May 2020; Revised 21 June 2020; Accepted 6 July 2020; Published 29 July 2020

Guest Editor: S. A. Edalatpanah

Copyright (C) 2020 Wadei F. Al-Omeri et al. This is an open access article distributed under the Creative Commons Attribution License, which permits unrestricted use, distribution, and reproduction in any medium, provided the original work is properly cited.

\begin{abstract}
In this manuscript, we obtain common fixed point theorems in the neutrosophic cone metric space. Also, notion of $(\Phi, \Psi)$-weak contraction is defined in the neutrosophic cone metric space by using the idea of altering distance function. Finally, we review many examples of cone metric spaces to verify some properties.
\end{abstract}

\section{Introduction}

The concept of fuzzy sets was introduced by Zadeh [1]. The study of fuzzy topological spaces was initiated by Chang [2]. The notion of intuitionistic fuzzy sets was introduced by Atanassov [3]. The notion of intuitionistic $L$-topological spaces was introduced by Atanassov and Stoeva [4] by extending $L$-topology to intuitionistic $L$-fuzzy setting. The notion of the intuitionistic fuzzy topological space was introduced by Çoker [5]. The concept of generalized fuzzy closed set was presented by Balasubramanian and Sundaram [6]. Smarandache extended the intuitionistic fuzzy sets to neutrosophic sets [7]. After the introduction of the neutrosophic set concept $[8,9]$ in 2019 by Smarandache and Shumrani on the nonstandard analysis, the nonstandard neutrosophic topology was developed. In recent years, neutrosophic algebraic structures have been investigated. Neutrosophy has laid the foundation for a whole family of new mathematical theories, generalizing both their classical and fuzzy counterparts, such as a neutrosophic theory in any field, see $[10,11]$. Recently, there were introduced neutrosophic mapping and neutrosophic connectedness. The concept of the neutrosophic metric space presented by [12] Al-Omeri et al. is a generalization of the intuitionistic fuzzy metric space due to Veeramani and George [13]. In 2019 and 2020, Smarandache generalized the classical Algebraic Structures to NeutroAlgebraic Structures (or NeutroAlgebras) whose operations and axioms are partially true, partially indeterminate, and partially false as extensions of Partial Algebra and to AntiAlgebraic Structures (or AntiAlgebras) whose operations and axioms are totally false. And in general, he extended any classical structure, in no matter what field of knowledge, to a NeutroStructure and an AntiStructure, see [14, 15]. In 2007, Huang and Zhang [16] introduced the concept of cone metric space and proved some fixed point theorems for contractive mappings. Recently, Öner et al. [17] introduced the concept of the fuzzy cone metric space that generalized the corresponding notions of the fuzzy metric space by George and Veeramani [13] and proved the fuzzy cone Banach contraction theorem. In 2010, Vetro et al. [18] extended the notion of $(\Phi, \Psi)$-weak contraction to fuzzy metric spaces and proved some common fixed point theorems for four mappings in fuzzy metric spaces by using the idea of an altering distance function. Gupta et al. and Wasfi et al. $[19,20]$ introduced the notions of E. A and common E. A on the modified intuitionistic generalized fuzzy metric space. They extended the notions of the common limit 
range property and E. A property for coupled maps on modified intuitionistic fuzzy metric spaces. This paper is devoted to the study of extending and generalizing the $(\Phi, \Psi)$-weak contraction to the neutrosophic cone metric space and prove some results. In Section 2, we will recall some materials which will be used throughout this paper. In Section 3, we give definitions and present the cone neutrosophic metric space and explain a number of properties. In Section 4, the results obtained from theorems and theoretical application of the neutrosophic fixed point are also presented. The last section contains the conclusions of the paper.

\section{Preliminaries}

Definition 1 (see [21]). Let $\Sigma$ be a non-empty fixed set. A neutrosophic set (briefly, NS) $R$ is an object having the form $R=\left\{\left\langle t, \xi_{R}(t), \varrho_{R}(t), \eta_{R}(t)\right\rangle: t \in \Sigma\right\}$, where $\xi_{R}(t), \varrho_{R}(t)$, and $\eta_{R}(t)$ which represent the degree of membership function (namely, $\xi_{R}(t)$ ), the degree of indeterminacy (namely, $\xi_{R}(t)$ ), and the degree of nonmembership (namely, $\eta_{R}(t)$ ), respectively, of each element $t \in \Gamma$ to the set $R$.

A neutrosophic set $H=\left\{\left\langle t, \xi_{H}(t), \varrho_{H}(t), \eta_{H}(t)\right\rangle: t \in \Gamma\right\}$ can be identified to an ordered triple $\left\langle\xi_{H}(t), \varrho_{H}(t), \eta_{H}(t)\right\rangle$ in $\left\lfloor 0^{-}, 1^{+}\right\rfloor$on $\Gamma$.

Remark 1 (see [21]). By using symbol $H=\left\{t, \xi_{H}(t), \varrho_{H}(t)\right.$, $\left.\eta_{H}(t)\right\}$ for the NS, $H=\left\{\left\langle t, \xi_{H}(t), \varrho_{H}(t), \eta_{H}(t)\right\rangle: t \in \Gamma\right\}$.

Definition 2 (see [13]). Let $H=\left\langle\xi_{H}(t), \varrho_{H}(t), \eta_{H}(t)\right\rangle$ be a NS on $\Gamma$. The complement of $H$ (briefly, $C(H)$ ) may be defined as three kinds of complements:

(1) $C(H)=\left\{\left\langle r, 1-\xi_{H}(t), 1-\eta_{H}(t)\right\rangle: t \in \Gamma\right\}$

(2) $C(H)=\left\{\left\langle r, \eta_{H}(t), 1-\varrho_{H}(t), \xi_{H}(t)\right\rangle: t \in \Gamma\right\}$

(3) $C(H)=\left\{\left\langle r, \eta_{H}(t), \varrho_{H}(t), \xi_{H}(t)\right\rangle: t \in \Gamma\right\}$

We have the following NSs (see [21]), which will be used in the sequel:

(1) $1_{N}=\{\langle t, 1,0,0\rangle: t \in \Gamma\}$ or

(2) $1_{N}=\{\langle t, 1,0,1\rangle: t \in \Gamma\}$,

(3) $1_{N}=\{\langle t, 1,1,0\rangle: t \in \Gamma\}$,

(4) $1_{N}=\{\langle t, 1,1,1\rangle: t \in \Gamma\}$.

(1) $0_{N}=\{\langle t, 0,1,1\rangle: t \in \Gamma\}$ or

(2) $0_{N}=\{\langle t, 0,0,1\rangle: t \in \Gamma\}$,

(3) $0_{N}=\{\langle t, 0,0,0\rangle: t \in \Gamma\}$,

(4) $0_{N}=\{\langle t, 0,1,0\rangle: t \in \Gamma\}$.

Definition 3 (see [21]). Let $\left\{H_{j}: j \in J\right\}$ be an arbitrary family of NSs in $\Gamma$. Then,

(1) $\cap H_{i}$ may be defined as follows:

$$
\begin{aligned}
& \text { (i) } \cap H_{i}=\left\langle t, \wedge \xi_{i \in \wedge} \xi_{H i}(t), \wedge_{i \in \wedge}^{\wedge} \varrho_{H i}(t), \bigvee_{i \in \wedge} \eta_{H i}(t)\right\rangle \\
& \text { (ii) } \cap H_{i}=\langle t, \wedge_{i \in \wedge}^{\wedge} \xi_{H i}(t), \bigvee_{i \in \wedge}^{\vee} \varrho_{H i}(t), \underbrace{}_{i \in \wedge} \eta_{H i}(t)\rangle
\end{aligned}
$$

(2) $\cup H_{i}$ may be defined as follows:

$$
\begin{aligned}
\text { (i) } \cup H_{i} & =\langle t, \vee_{i \in \wedge} \xi_{H i}(t), \underbrace{\vee}_{i \in \wedge} \varrho_{H i}(t), \wedge_{i \in \wedge}^{\wedge} \eta_{H i}(t)\rangle \\
\text { (ii) } \cup H_{i} & =\left\langle t, \bigvee_{i \in \wedge} \xi_{H i}(t), \bigwedge_{i \in \wedge}^{\wedge} \varrho_{H i}(t), \bigwedge_{i \in \wedge}^{\wedge} \eta_{H i}(t)\right\rangle
\end{aligned}
$$

Definition 4 (see [21]). For any $r \neq \varnothing$, let neutrosophic sets $R$ and $\Gamma$ be in the form $R=\left\{r, \xi_{R}(r), \varrho_{R}(r), \eta_{R}(r)\right\}$ and $\Gamma=\left\{r, \xi_{\Gamma}(r), \varrho_{\Gamma}(r), \eta_{\Gamma}(r)\right\}$. The two possible definitions of $R \subseteq \Gamma$ are as follows:

$$
\begin{aligned}
\text { (1) } & R \subseteq \Gamma \Longleftrightarrow \xi_{R}(r) \leq \xi_{\Gamma}(r), \varrho_{R}(r) \geq \varrho_{\Gamma}(r) \text {, and } \eta_{R}(r) \\
& \leq \eta_{\Gamma}(r) \\
\text { (2) } & R \subseteq \Gamma \Longleftrightarrow \xi_{R}(r) \leq \xi_{\Gamma}(r), \varrho_{R}(r) \geq \varrho_{\Gamma}(r) \text {, and } \eta_{R}(r) \\
& \geq \eta_{\Gamma}(r)
\end{aligned}
$$

Definition 5 (see [22]). A neutrosophic topology (NT for short) and a nonempty set $\Gamma$ is a family $\Xi$ of neutrosophic subsets in $\Gamma$ satisfying the following axioms:
(1) $0_{N}, 1_{N} \in \Xi$
(2) $B_{1} \cap B_{2} \in \Xi$ for any $B_{1}, B_{2} \in \Xi$
(3) $\cup B_{i} \in \Xi, \forall\left\{B_{i} \mid i \in I\right\} \subseteq \Xi$

The elements of $\Xi$ are called open neutrosophic sets. The pair $(\Gamma, \Xi)$ is called a neutrosophic topological space, and any neutrosophic set in $\Xi$ is known as the neutrosophic open set (NOS) in $\Gamma$. A neutrosophic set $B$ is closed if its complement is neutrosophic-open, denoted by $C(B)$. Throughout this paper, we suppose that all cone metrics have nonempty interior.

For any NTS $R$ in $(\Gamma, \Xi) \quad[23]$, we have $C l\left(R^{c}\right)=[\operatorname{Int}(R)]^{c}$ and $\operatorname{Int}\left(R^{c}\right)=[C l(R)]^{c}$.

Definition 6. A subset $\mu$ of $\Sigma$ is said to be a cone in the following cases:

(1) If both $s \in \mu$ and $-s \in \mu$, then $s=\phi$

(2) If $s, r \in S, s, r \geq 0$, and $u, v \in \mu$, then $s u+r v \in \mu$

(3) $\mu$ is closed, nonempty, and $\mu \neq\{\phi\}$

For a given cone, partial ordering $(\preccurlyeq)$ on $\Sigma$ via $\mu$ is defined by $u \leqslant v$ iff $v-u \in \mu$. $u \prec v$ will stand for $u \leqslant v$ and $u \ll v$, while $u \neq v$ will stand for $v-u \in \operatorname{Int}(\mu)$.

If $\exists$ a constant $K>0$ such that for all $\varnothing \preccurlyeq u \preccurlyeq v, u, v \in \Sigma$ implies $\|u\| \leq K\|v\|$, and the least positive number $K$ satisfying this property is called the normal constant of $P$, where $P$ is the normal.

Definition 7. Let $\Gamma$ be a nonempty set and $s \geq 1$ be a given real number. A function $d: \Gamma \times \Gamma \mapsto \Sigma$ is said to be a cone metric on $\Gamma$ if the following conditions hold:

(1) $d\left(m_{1}, m_{2}\right)=d\left(m_{2}, m_{1}\right)$ for all $m_{1}, m_{2} \in \Gamma$

(2) $0 \preccurlyeq d\left(m_{1}, m_{2}\right)$ for all $m_{1}, m_{2} \in \Gamma$

(3)

$d\left(m_{1}, m_{3}\right) \leqslant s\left(d\left(m_{1}, m_{2}\right)+d\left(m_{2}, m_{3}\right)\right) \forall m_{1}, m_{2}, m_{3} \in \Gamma$

(4) $d\left(m_{1}, m_{2}\right)=0$ iff $m_{1}=m_{2}$ CMS).

The pair $(\Gamma, d)$ is called a cone metric space (shortly, 
Definition 8. A $t$-norm is continuous for any binary operation $*:[0,1] \times[0,1] \longrightarrow[0,1]$ if $*$ verifies the following statements:

(1) $*$ is continuous

(2) $*$ is commutative and associative

(3) $n_{1} * n_{2} \leq n_{3} * n_{4}$ whenever $n_{1} \leq n_{3}$ and $n_{2} \leq n_{4}$ for all $n_{1}, n_{2}, n_{3}, n_{4} \in[0,1]$

(4) $n_{1} * 1=n_{1}$ for all $n_{1} \in[0,1]$

Definition 9. Let $(\Gamma, d)$ be a CMS. Then, for any $d_{1} \gg 0$ and $d_{2} \gg 0, d_{1}, d_{2} \in \Sigma, \exists d \gg 0$, and $d \in \Sigma$ such that $d \ll d_{1}$ and $d \ll d_{2}$.

Example 1. $n_{1} * n_{2}=\max \left\{n_{1}, n_{2}\right\}$ and $n_{1} * n_{2}=n_{1} n_{2}$.

Example 2. $n_{1} \diamond n_{2}=\max \left\{n_{1}, n_{2}\right\}$ and $m_{1} \diamond n_{2}=\min \left\{n_{1}+\right.$ $\left.n_{2}, 1\right\}$.

Definition 10. A $t$-conorm of a binary operation $\diamond$ : $[0,1] \times$ $[0,1] \longrightarrow[0,1]$ is continuous if $\diamond$ verifies the following statements:

$(1) \diamond$ is continuous

(2) $\diamond$ is associative and commutative

(3) $q_{1} \diamond q_{2} \leq q_{3} \diamond q_{4}$ whenever $q_{1} \leq q_{3}$ and $q_{2} \leq q_{4}$ for all $q_{1}, q_{2}, q_{3}, q_{4} \in[0,1]$

(4) $q_{1} \diamond 1=q_{1}$ for all $q_{1} \in[0,1]$

Definition 11 (see [12]). ( $\Gamma, \psi, \phi, *, \diamond)$ is said to be a neutrosophic cone metric space if $\mu$ is NCMS of $\Sigma, \Gamma$ is an arbitrary set, $\diamond$ is a $\mathrm{N}$-continuous $t$-conorm, $*$ is a $\mathrm{N}$-continuous $t$-norm, and $\psi, \phi$ are neutrosophic sets on $\Gamma^{3} \times \operatorname{Int}(\mu)$, which satisfy the following statements: $\forall \varepsilon_{1}, \varepsilon_{2}, \varepsilon_{3} \in \Gamma$ and $n, m \in \operatorname{Int}(\mu)$ (that is, $n \gg 0_{\phi}$ and $m \gg 0_{\phi}$ ):

(1) $\psi\left(\varepsilon_{1}, \varepsilon_{2}, \varepsilon_{3}, m\right)>0_{\phi} \forall \varepsilon_{1}, \varepsilon_{2}, \varepsilon_{3} \in \Gamma$

(2) $\psi\left(\varepsilon_{1}, \varepsilon_{2}, \varepsilon_{3}, m\right)=1$ iff $\varepsilon_{1}=\varepsilon_{2}=\varepsilon_{3}$

(3) $\psi\left(\varepsilon_{1}, \varepsilon_{2}, \varepsilon_{3}, m\right)=\psi\left(p\left\{\varepsilon_{1}, \varepsilon_{2}, \varepsilon_{3}\right\}, m\right)$, where $p$ is permutation

(4) $\psi\left(\varepsilon_{1}, \varepsilon_{2}, \varepsilon_{3}, m\right) * \psi\left(\varepsilon_{2}, \varepsilon_{3}, n\right) \leq \psi\left(\varepsilon_{1}, \varepsilon_{3}, m+n\right)$

(5) $\left.\psi\left(\varepsilon_{1}, \varepsilon_{2}, \varepsilon_{3},.\right): \operatorname{Int}(\mu) \longrightarrow\right\rfloor 0^{-}, 1^{+}\lfloor$is neutrosophiccontinuous

Definition 12 (see [12]). Let $(\Gamma, \psi, \phi, *, \diamond)$ be a NCMS. For $m \gg 0_{\phi}$, the open ball $\Gamma(x, s, m)$ with center $\varepsilon_{1}$ and radius $s \in(0,1)$ is defined by $\left(\varepsilon_{1}, s, m\right)=\left\{\varepsilon_{2} \in \Gamma: \psi\left(\varepsilon_{1}, \varepsilon_{2}, m\right)\right.$ $\left.>1-m, \phi\left(\varepsilon_{1}, \varepsilon_{2}, m\right)<s\right\}$.

Example 3. Let $\Sigma=R^{+}$. Then, $\mu=\left\{\left(p_{1}, p_{2}, p_{3}\right)\right.$ : $\left.p_{1}, p_{2}, p_{3} \geq 0\right\} \subseteq \Sigma$ is a normal cone, and $P=1$ is a normal constant. Let $s * t=s t, \Gamma=R$, and $\psi: \Gamma^{3} \times \operatorname{int}(\mu) \longrightarrow[0,1]$, defined by $\psi\left(\varepsilon_{1}, \varepsilon_{2}, \varepsilon_{3}, t\right)=\left(1 / e^{\left(\left|\varepsilon_{1}-\varepsilon_{2}\right|+\left|\varepsilon_{2}-\varepsilon_{3}\right|+\left|\varepsilon_{3}-\varepsilon_{1}\right| /\|t\|\right)}\right)$ $\forall \varepsilon_{1}, \varepsilon_{2}, \varepsilon_{3} \in \Gamma$ and $t \gg \varnothing$.
Definition 13 (see [12]). An $(\Gamma, \psi, \phi, *, \diamond)$ neutrosophic cone metric is called complete neutrosophic if any sequence which is Cauchy in $\operatorname{NCMS}(\Gamma, \psi, \phi)$ is convergent.

Definition 14 (see [12]). ( $\Gamma, \psi, \phi, *, \diamond)$ is said to be a neutrosophic CMS if $\mu$ is a neutrosophic cone metric (shortly, NCMS) of $\Sigma$, where $\Gamma$ is an arbitrary set, $*$ is a neutrosophic continuous $t$-norm, $\diamond$ is a neutrosophic continuous $t$ conorm, and $\psi, \phi$ are neutrosophic sets on $\Gamma^{3} \times \operatorname{Int}(\mu)$, which satisfy the following statements: $\forall \varepsilon_{1}, \varepsilon_{2}, \varepsilon_{3} \in \Gamma$ and $m, n \in \operatorname{Int}(\mu)$ (that is, $n \gg 0_{\phi}$ and $m \gg 0_{\phi}$ ):

(1) $\psi\left(\varepsilon_{1}, \varepsilon_{2}, \varepsilon_{3}, m\right)=1$ iff $\varepsilon_{1}=\varepsilon_{2}=\varepsilon_{3}$

(2) $\psi\left(\varepsilon_{1}, \varepsilon_{2}, \varepsilon_{3}, m\right) * \psi\left(\varepsilon_{2}, \varepsilon_{3}, n\right) \leq \psi\left(\varepsilon_{1}, \varepsilon_{3}, n+m\right)$

(3) $\psi\left(\varepsilon_{1}, \varepsilon_{2}, \varepsilon_{3}, m\right)=\psi\left(p\left\{\varepsilon_{1}, \varepsilon_{2}, \varepsilon_{3}\right\}, m\right)$, where $p$ is permutation

(4) $\psi\left(\varepsilon_{1}, \varepsilon_{2}, \varepsilon_{3}, m\right)+\phi\left(\varepsilon_{1}, \varepsilon_{2}, \varepsilon_{3}\right) \leq 1_{\phi}$

(5) $\left.\psi\left(\varepsilon_{1}, \varepsilon_{2}, \varepsilon_{3},.\right): \operatorname{Int}(\mu) \longrightarrow\right\rfloor 0^{-}, 1^{+}\lfloor$is neutrosophiccontinuous

(6) $\phi\left(\varepsilon_{1}, \varepsilon_{2}, \varepsilon_{3}, m\right) \diamond \phi\left(\varepsilon_{2}, \varepsilon_{3}, n\right) \geq \phi\left(\varepsilon_{1}, \varepsilon_{3}, m+n\right)$

(7) $\left.\phi\left(\varepsilon_{1}, \varepsilon_{2}, \varepsilon_{3},.\right): \operatorname{Int}(\mu) \diamond\right\rfloor 0^{-}, 1^{+}\lfloor$is neutrosophiccontinuous

(8) $\phi\left(\varepsilon_{1}, \varepsilon_{2}, \varepsilon_{3}, m\right)<0_{\phi}$

(9) $\phi\left(\varepsilon_{1}, \varepsilon_{2}, \varepsilon_{3}, m\right)=0_{\phi}$ if and only if $\varepsilon_{1}=\varepsilon_{2}=\varepsilon_{3}$

(10) $\phi\left(\varepsilon_{1}, \varepsilon_{2}, \varepsilon_{3}, m\right)>0_{\phi} \forall \varepsilon_{1}, \varepsilon_{2}, \varepsilon_{3} \in \Gamma$

(11) $\phi\left(\varepsilon_{1}, \varepsilon_{2}, \varepsilon_{3}, m\right)=\phi\left(p\left\{\varepsilon_{1}, \varepsilon_{2}, \varepsilon_{3}\right\}, m\right)$, where $p$ is permutation

Then, $(\psi, \phi)$ is called a neutrosophic cone metric on $\Gamma$.

The functions $\psi\left(\varepsilon_{1}, \varepsilon_{2}, m\right)$ and $\phi\left(\varepsilon_{1}, \varepsilon_{2}, m\right)$ are defined by the degree of non-nearness between $\varepsilon_{1}$ and $\varepsilon_{2}$ with respect to $m$, respectively.

Definition 15 (see [12]). Let $(\Gamma, \psi, \phi, *, \diamond)$ be a NCMS, $\varepsilon_{1} \in \Gamma$, and $\left\{\varepsilon_{1 n}\right\}$ be a sequence in $\Gamma$. Then, $\left\{\varepsilon_{1 n}\right\}$ is said to be convergent to $\varepsilon_{1}$ if for all $m \gg 0_{\phi}$ and all $s \in(0,1)$, there exists $n_{0} \in N$ such that $\psi\left(\varepsilon_{1 n}, \varepsilon_{1}, m\right)>1-s, \phi\left(\varepsilon_{1 n}, \varepsilon_{1}, m\right) \leq s$ for any $n \geq n_{0}$. We defined that $\lim _{n \rightarrow \infty} \varepsilon_{1 n}=\varepsilon_{1}$ or $\varepsilon_{1 n} \longrightarrow \varepsilon_{1}$ as $n \longrightarrow \infty$.

Definition 16. A function $\Phi:[0, \infty) \longrightarrow[0, \infty)$ is an altering distance if $\Phi(n)$ is monotone increasing and continuous, and $\Phi(n)=0$ iff $n=\varnothing$.

Definition 17. Let $(\Gamma, d)$ be a metric space and let $\Sigma=R^{+}$. Defined $\mu_{1} \diamond \mu_{2}=\min \left\{\mu_{1}+\mu_{2}, 1\right\}$ and $\mu_{1} * \mu_{1}=\mu_{1} \mu_{2}$ for any $\mu_{1}, \mu_{2} \in[0,1]$, and let $\Gamma$ and $\psi$ be fuzzy sets on $\Gamma^{3} \times \operatorname{int}(\mu)$ represented by $\psi\left(\varepsilon_{1}, \varepsilon_{2}, \varepsilon_{3}, \mu\right)=\left(k t^{n} / k t^{n}+\mathscr{L} D *\left(\varepsilon_{1}, \varepsilon_{2}, \varepsilon_{3}\right)\right)$ and $\phi\left(\varepsilon_{1}, \varepsilon_{2}, \varepsilon_{3}, \mu\right)=\left(D *\left(\varepsilon_{1}, \varepsilon_{2}, \varepsilon_{3}\right) / m t^{n}+\mathscr{L} D *\left(\varepsilon_{1}, \varepsilon_{2}\right.\right.$, $\left.\left.\varepsilon_{3}\right)\right)$

\section{Main Result}

Definition 18. Let $(\Gamma, \psi, \phi, *, \diamond)$ be a neutrosophic cone metric space (CMS) and $\mathscr{T}, \mathscr{H}: \Gamma \longrightarrow \Gamma$ be two mappings. Mapping $\mathscr{H}$ is said to be neutrosophic $(\Phi, \Psi)$-weak contraction if there exists a function $\Psi:[0, \infty) \longrightarrow[0, \infty)$ with 
$\Psi(s)>0$ and $\Psi(s)=0$ for $s>0$ and an alternating distance function $\Phi$ such that

$$
\begin{gathered}
\Phi\left(\frac{1}{\psi\left(\mathscr{H}\left(\varepsilon_{1}\right), \mathscr{H}\left(\varepsilon_{2}\right), \mathscr{H}\left(\varepsilon_{3}\right), m\right)}-1_{\phi}\right) \leq \Phi\left(\frac{1}{\psi\left(\mathscr{T}\left(\varepsilon_{1}\right), \mathscr{T}\left(\varepsilon_{2}\right), \mathscr{T}\left(\varepsilon_{3}\right), m\right)}-1_{\phi}\right)-\Psi\left(\frac{1}{\psi\left(\mathscr{T}\left(\varepsilon_{1}\right), \mathscr{T}\left(\varepsilon_{2}\right), \mathscr{T}\left(\varepsilon_{3}\right), m\right)}-1_{\phi}\right) \\
\Phi\left(\phi\left(\mathscr{H}\left(\varepsilon_{1}\right), \mathscr{H}\left(\varepsilon_{2}\right), \mathscr{H}\left(\varepsilon_{3}\right), m\right)\right) \leq \Phi\left(\phi\left(\mathscr{T}\left(\varepsilon_{1}\right), \mathscr{T}\left(\varepsilon_{2}\right), \mathscr{T}\left(\varepsilon_{3}\right), m\right)\right)-\Psi\left(\phi\left(\mathscr{T}\left(\varepsilon_{1}\right), \mathscr{T}\left(\varepsilon_{2}\right), \mathscr{T}\left(\varepsilon_{3}\right), m\right)\right) .
\end{gathered}
$$

hold for all $\varepsilon_{1}, \varepsilon_{2}, \varepsilon_{3} \in \psi$ and each $m \gg 0_{\phi}$. If $\mathscr{T}$ is the identity map, then $\mathscr{H}$ is called a neutrosophic $(\Phi, \Psi)$-weak contraction mapping.

Definition 19. Let $(\Gamma, \psi, \phi, *, \diamond)$ be a neutrosophic cone metric space and $\mathscr{T}, \mathscr{H}: \Gamma \longrightarrow \Gamma$ be two mappings. Point $v$ is said to be a coincidence point in $\psi$ of $\mathscr{T}$ and $\mathscr{H}$ if $\varepsilon_{3}=\mathscr{T}(v)=\mathscr{H}(v)$.

Definition 20. Let $\left\{\mathscr{T}_{i}\right\}$ and $\left\{\mathscr{H}_{i}\right\}$ be two finite families of self-mappings on $\psi$. They are called pairwise commuting if

(1) $\mathscr{T}_{i} \mathscr{T}_{j}=\mathscr{T}_{j} \mathscr{T}_{i}$, where $i, j \in\{1,2, \ldots, n\}$

(2) $\mathscr{H}_{i} \mathscr{H}_{j}=\mathscr{H}_{j} \mathscr{H}_{i}$, where $i, j \in\{1,2, \ldots, m\}$

(3) $\mathscr{T}_{i} \mathscr{H}_{j}=\mathscr{H}_{j} \mathscr{T}_{i}$, where $i \in\{1,2, \ldots, n\} \quad$ and $j \in\{1,2, \ldots, m\}$
Theorem 1. Let $(\Gamma, \psi, \phi, *, \diamond)$ be a neutrosophic cone metric space and $\mathscr{H}: \Gamma \longrightarrow \Gamma$ be a neutrosophic $(\Phi, \Psi)$-weak contraction with respect to $\mathscr{T}: \Gamma \longrightarrow \Gamma$. If $\mathscr{H}(\psi) \subseteq \mathscr{T}(\psi)$ and $\mathscr{T}(\psi)$ or $\mathscr{H}(\psi)$ is a complete subset of $\psi$, then $\mathscr{T}$ and $\mathscr{H}$ have a unique common fixed point in $\psi$ provided that $\Psi$ is a continuous function.

Proof. Let $t_{0} \in \psi$ be an arbitrary point. Let point $t_{1} \in \psi$ such that $\mathscr{H}\left(t_{0}\right)=\mathscr{T}\left(t_{1}\right)$. This can be done since $\mathscr{H}(\psi) \subseteq \mathscr{T}(\psi)$. Continuing this process, we obtain a sequence $\left\{t_{n}\right\} \in \psi$ such that $s_{n}=\mathscr{H}\left(t_{n}\right)=\mathscr{T}\left(t_{n+1}\right)$. We assume that $s_{n} \neq s_{n+1}$ for all $n \in \mathbb{N}$; otherwise, $\mathscr{T}$ and $\mathscr{H}$ have a coincidence point. Now, we get

$$
\begin{aligned}
\Phi\left(\frac{1}{\psi\left(s_{n}, s_{n}, s_{n+1}, m\right)}-1_{\phi}\right)= & \Phi\left(\frac{1}{\psi\left(\mathscr{H}\left(t_{n}\right), \mathscr{H}\left(t_{n}\right), \mathscr{H}\left(t_{n+1}\right), m\right)}-1_{\phi}\right) \\
\leq & \Phi\left(\frac{1}{\psi\left(\mathscr{T}\left(t_{n}\right), \mathscr{T}\left(t_{n}\right), \mathscr{T}\left(t_{n+1}\right), m\right)}-1_{\phi}\right) \\
& -\Psi\left(\frac{1}{\psi\left(\mathscr{T}\left(t_{n}\right), \mathscr{T}\left(t_{n}\right), \mathscr{T}\left(t_{n+1}\right), m\right)}-1_{\phi}\right) \\
\leq & \Phi\left(\frac{1}{\psi\left(s_{n-1}, s_{n-1}, s_{n}, m\right)}-1_{\phi}\right) \\
& -\Psi\left(\frac{1}{\psi\left(s_{n-1}, s_{n-1}, s_{n}, m\right)}-1_{\phi}\right) \\
\leq & \left(\frac{1}{\psi\left(s_{n-1}, s_{n-1}, s_{n}, m\right)}-1_{\phi}\right)
\end{aligned}
$$

which suppose that $\mathscr{T}$ mapping is nondecreasing; hence, $\psi\left(s_{n}, s_{n}, s_{n+1}, m\right)>\psi\left(s_{n-1}, s_{n-1}, s_{n}, m\right) \forall n \in \mathbb{N}$. Hence, $\psi\left(s_{n-1}, s_{n-1}, s_{n}, m\right)$ is an increasing sequence of positive real numbers in $(0,1]$. Let $V(m)=\lim _{n \longrightarrow \infty} \psi\left(s_{n-1}, s_{n-1}, s_{n}, m\right)$. We prove that $V(m)=1 \forall m \gg 0_{\phi}$. If not, there exists $m \gg 0_{\phi}$ such that $V(m)<1_{\phi}$. Then, from the above inequality on taking $n \longrightarrow \infty$, we obtain

which is a contradiction. Therefore, $\psi\left(s_{n}, s_{n}, s_{n+1}, m\right) \longrightarrow 1$ as $n \longrightarrow \infty$. Now, for each $k \geq 0$, by Definition 18 , we get 


$$
\begin{gathered}
\psi\left(s_{n}, s_{n}, s_{n+k}, m\right) \geq \psi\left(s_{n}, s_{n}, s_{n+1}, \frac{m}{k}\right) * \psi\left(s_{n+1}, s_{n+1}, s_{n+2}, \frac{m}{k}\right) \\
* \cdots * \psi\left(s_{n+k-1}, s_{n+k-1}, s_{n+k}, \frac{m}{k}\right) .
\end{gathered}
$$

It follows that $\lim _{n \longrightarrow \infty} \psi\left(s_{n}, s_{n}, s_{n+k}, m\right) \geq 1 * 1 * \cdots * 1$ $=1$. At the same time, we have

$$
\begin{aligned}
\Phi\left(\phi\left(s_{n}, s_{n}, s_{n+1}, m\right)\right)= & \Phi\left(\phi\left(\mathscr{H}\left(t_{n}\right), \mathscr{H}\left(t_{n}\right), \mathscr{H}\left(t_{n+1}\right), m\right)\right) \\
\leq & \Phi\left(\phi\left(\mathscr{T}\left(t_{n}\right), \mathscr{T}\left(t_{n}\right), \mathscr{T}\left(t_{n+1}\right), m\right)\right) \\
& -\Psi\left(\phi\left(\mathscr{T}\left(t_{n}\right), \mathscr{T}\left(t_{n}\right), \mathscr{T}\left(t_{n+1}\right), m\right)\right) \\
\leq & \Phi\left(\phi\left(s_{n-1}, s_{n-1}, s_{n}, m\right)\right) \\
& -\Psi\left(\phi\left(s_{n-1}, s_{n-1}, s_{n}, m\right)\right) \\
< & \Phi\left(\phi\left(s_{n-1}, s_{n-1}, s_{n}, m\right)\right) .
\end{aligned}
$$

in which considering that the $\mathscr{T}$ mapping is nondecreasing, then $\phi\left(s_{n}, s_{n}, s_{n+1}, m\right)<\phi\left(s_{n-1}, s_{n-1}, s_{n}, m\right) \forall n \in \mathbb{N}$. Thus, $\phi\left(s_{n-1}, s_{n-1}, s_{n}, m\right)$ is a decreasing sequence of positive real numbers in $[0,1)$. Let $U(m)=\lim _{n \longrightarrow \infty} \phi\left(s_{n-1}, s_{n-1}, s_{n}, m\right)$. We show that $U(m)=0_{\phi}$ for all $m \gg 0_{\phi}$. If this is not the case, there exists $m \gg 0_{\phi}$ such that $U(m)>0_{\phi}$. Then, it follows from (5), by taking $n \longrightarrow \infty$, that $\Phi(U(m)) \leq \Phi(U(m))-\Psi(U(m))$, which is a contraction. Therefore, $\phi\left(s_{n}, s_{n}, s_{n+1}, m\right) \longrightarrow 0_{\phi}$ as $n \longrightarrow \infty$. Now, for each $k \geq 0$, by Definition 14 (9), we have

$$
\begin{array}{r}
\psi\left(s_{n}, s_{n}, s_{n+k}, m\right)+\phi\left(s_{n}, s_{n}, s_{n+k}, m\right) \leq 1_{\phi}, \\
\lim _{n \longrightarrow \infty}\left[\psi\left(s_{n}, s_{n}, s_{n+k}, m\right)+\phi\left(s_{n}, s_{n}, s_{n+k}, m\right)\right] \leq 1_{\phi} .
\end{array}
$$

It follows that $\lim _{n \longrightarrow \infty} \phi\left(s_{n}, s_{n}, s_{n+k}, m\right)=0_{\phi}$. Hence, $s_{n}$ is a Cauchy sequence. If $\mathscr{T}(\psi)$ is complete, then there exists $k \in \mathscr{T}(\psi)$ such that $s_{n} \longrightarrow k$ as $n \longrightarrow \infty$. The same holds if $\mathscr{H}(\psi)$ is complete with $k \in \mathscr{H}(\psi)$. Let $k \in \psi$ and $\mathscr{T}(k)=p$. Now, we shall show that $k$ is a coincidence point of $\mathscr{T}$ and $\mathscr{H}$. In fact, we have taken

$$
\begin{aligned}
\Phi\left(\frac{1}{\psi\left(\mathscr{H}(k), \mathscr{H}(k), \mathscr{T}\left(t_{n+1}\right), m\right)}-1_{\phi}\right) & =\Phi\left(\frac{1}{\psi\left(\mathscr{H}(k), \mathscr{H}(k), \mathscr{H}\left(t_{n}\right), m\right)}-1_{\phi}\right) \\
\leq & \Phi\left(\frac{1}{\psi\left(\mathscr{T}(k), \mathscr{T}(k), \mathscr{T}\left(t_{n}\right), m\right)}-1_{\phi}\right) \\
& -\Psi\left(\frac{1}{\psi\left(\mathscr{T}(k), \mathscr{T}(k), \mathscr{T}\left(t_{n}\right), m\right)}-1_{\phi}\right),
\end{aligned}
$$

for every $m \gg 0_{\phi}$, in which by letting $n \longrightarrow \infty$,

$$
\begin{aligned}
\lim _{n \longrightarrow \infty} \psi\left(\mathscr{H}(k), \mathscr{H}(k), \mathscr{T}\left(t_{n+1}\right), m\right) \\
\quad=\lim _{n \longrightarrow \infty} \psi\left(\mathscr{H}(k), \mathscr{H}(k), \mathscr{H}\left(t_{n}\right), m\right) \\
=\psi(\mathscr{H}(k), \mathscr{H}(k), \mathscr{T}(k), m) \\
=1 .
\end{aligned}
$$

Therefore, $\mathscr{T}(k)=\mathscr{H}(k)=p$. Now, we shall prove that $\mathscr{T}(p)=p$. If it is not so, then we have

$$
\begin{aligned}
\Phi\left(\frac{1}{\psi(\mathscr{T}(p), \mathscr{T}(p), \mathscr{T}(p), m)}-1_{\phi}\right)= & \Phi\left(\frac{1}{\psi(\mathscr{H}(p), \mathscr{H}(p), \mathscr{H}(k), m)}-1_{\phi}\right) \\
\leq & \Phi\left(\frac{1}{\psi(\mathscr{T}(p), \mathscr{T}(p), \mathscr{T}(k), m)}-1_{\phi}\right) \\
& -\Psi\left(\frac{1}{\psi(\mathscr{T}(p), \mathscr{T}(p), \mathscr{T}(k), m)}-1_{\phi}\right) \\
\leq & \Phi\left(\frac{1}{\psi(\mathscr{T}(p), \mathscr{T}(p), p, m)}-1_{\phi}\right) \\
& -\Psi\left(\frac{1}{\psi(\mathscr{T}(p), \mathscr{T}(p), p, m)}-1_{\phi}\right),
\end{aligned}
$$


which is a contradiction. By inequalities (4) and (5) we prove the uniqueness. The desired equality is obtained.

Example 4. Let $(\Gamma, \psi, \phi, *, \diamond)$ be a complete neutrosophic cone metric space, $\Gamma=\{(1 / n): n \in \mathbb{N}\} \cup 0_{\phi}, \diamond$ be a maximum norm, and $*$ be a minimum norm. Let $\psi, \phi$ be defined by

$$
\begin{aligned}
& \psi\left(\varepsilon_{1}, \varepsilon_{2}, \varepsilon_{3}, m\right)= \begin{cases}\frac{m}{m+(|t+s|+|s+r|+|r+t|)}, & \text { if } m>0_{\phi}, \\
0, & \text { if } m=0_{\phi},\end{cases} \\
& \phi\left(\varepsilon_{1}, \varepsilon_{2}, \varepsilon_{3}, m\right)= \begin{cases}\frac{|t+s|+|s+r|+|r+t|}{m+(|t+s|+|s+r|+|r+t|)}, & \text { if } m>0_{\phi}, \\
0, & \text { if } m=0_{\phi} .\end{cases}
\end{aligned}
$$

Also, define $(\Phi, \Psi):[0, \infty) \longrightarrow[0, \infty)$ by $\mathscr{T}(t)=(t / 2)$, and $\mathscr{H}(t)=(t / 4)$. Obviously, $\mathscr{H}(\Gamma) \subseteq \mathscr{T}(\Gamma)$, $\Phi(m)=(m / 2)$ and $\Psi(m)=(m / 8)$, for all $m \gg p$, and $\Psi$ is a continuous function. Then, we have

$$
\begin{aligned}
& \Phi\left(\frac{1}{\psi(\mathscr{T}(t), \mathscr{T}(s), \mathscr{T}(r), m)}-1_{\phi}\right)-\Psi\left(\frac{1}{\psi(\mathscr{T}(t), \mathscr{T}(s), \mathscr{T}(r), m)}-1_{\phi}\right) \\
& \quad=\frac{3(|t+s|+|s+r|+|r+t|)}{16 m} \\
& \quad \geq \frac{2(|t+s|+|s+r|+|r+t|)}{16 m} \\
& \quad=\Phi\left(\frac{1}{\psi(\mathscr{H}(t), \mathscr{H}(s), \mathscr{H}(r), m)}-1_{\phi}\right) .
\end{aligned}
$$

From the above inequality and the fact that $\phi=1_{\phi}-\psi$, we conclude that the conditions (1) and (2) in Definition 2.18 are satisfied. Thus, $\mathscr{H}$ is a neutrosophic $(\Phi-\Psi)$-weak contraction with respect to $\mathscr{T}$.

Corollary 1. Let $(\Gamma, \psi, \phi, *, \diamond)$ be a neutrosophic cone metric space and $\mathscr{H}: \Gamma \longrightarrow \Gamma$ be a neutrosophic $(\Phi, \Psi)$-weak contraction. If $\Psi$ is continuous, then $\mathscr{H}$ has a unique fixed point.

Corollary 2. Let $(\Gamma, \psi, \phi, *, \diamond)$ be a neutrosophic cone metric space. Then, $\mathscr{H}: \Gamma \diamond \Gamma$ is a mapping satisfying

$$
\begin{gathered}
\Phi\left(\frac{1}{\psi(\mathscr{H}(t), \mathscr{H}(s), \mathscr{H}(r), m)}-1_{\phi}\right) \leq p \Phi\left(\frac{1}{\psi(t, s, r, m)}-1_{\phi}\right), \\
\Phi(\phi(\mathscr{H}(t), \mathscr{H}(s), \mathscr{H}(r), m)) \leq p \Phi(\phi(t, s, r, m)) .
\end{gathered}
$$

for each $t, s, r \in \Gamma, m \gg 0_{\phi}$, and $p \in(0,1)$.

Theorem 2. Let $(\Gamma, \psi, \phi, *, \diamond)$ be a neutrosophic cone metric space and $\mathscr{T}_{j}, \mathscr{H}_{i}$ be two finite self-mappings on $\Gamma$ with $\mathscr{T}=$ $\mathscr{T}_{1} \cdot \mathscr{T}_{2} \ldots \mathscr{T}_{m}$ and $\mathscr{H}=\mathscr{H}_{1} \cdot \mathscr{H}_{2} \ldots \mathscr{H}_{n}$ such that $i \in\{1,2, \ldots, n\}$ and $j \in\{1,2, \ldots, m\}$. Suppose $\mathscr{H}$ be a generalized neutrosophic $(\Phi, \Psi)$-weak contraction which is given with respect to $\mathscr{T}$. If $\mathscr{T}(\Gamma)$ and $\mathscr{H}(\Gamma) \subseteq \mathscr{T}(\Gamma)$ or $\mathscr{H}(\Gamma)$ is a complete subset of $\Gamma$, then $\mathscr{H}_{i}, \mathscr{T}_{j}$ have a common fixed point in which $\Gamma$ is unique, provided a description of $\Psi$ is a continuous function and the families $\mathscr{T}_{j}, \mathscr{H}_{i}$ commute pairwise.

Proof. By Theorem 1, we obtain that $\mathscr{T}$ and $\mathscr{H}$ have a common fixed point that is unique, say $p$. In order to prove that $p$ remains as a fixed point of all self-mappings, let

$$
\begin{aligned}
\mathscr{H}_{j} \mathscr{H}_{j}(p) & =\left(\mathscr{H}_{1} \mathscr{H}_{2} \ldots \mathscr{H}_{n}\right) \mathscr{H}_{j}(p) \\
& =\left(\mathscr{H}_{1} \mathscr{H}_{2} \ldots \mathscr{H}_{n-1}\right) \mathscr{H}_{n} \mathscr{H}_{j}(p) \\
& =\left(\mathscr{H}_{1} \mathscr{H}_{2} \ldots \mathscr{H}_{n-1}\right) \mathscr{H}_{j} \mathscr{H}_{n}(p) \\
& =\ldots \\
& =\mathscr{H}_{1} \mathscr{H}_{j}\left(\mathscr{H}_{2} \mathscr{H}_{3} \ldots \mathscr{H}_{n}\right)(p) \\
& =\mathscr{H}_{j} \mathscr{H}_{1}\left(\mathscr{H}_{2} \mathscr{H}_{3} \ldots \mathscr{H}_{n}\right)(p) \\
& =\mathscr{H}_{j} \mathscr{H}_{(p)} \\
& =\mathscr{H}_{j}(p) .
\end{aligned}
$$


Since the other conditions are similarly proved, we can show that $\mathscr{H}_{\mathcal{T}_{i}}(p)=\mathscr{T}_{i} \mathscr{H}(p)=\mathscr{T}_{i}(p), \quad \mathscr{T} \mathscr{T}_{i}(p)=$ $\mathscr{T}_{i} \mathscr{T}(p)=\mathscr{T}_{i}(p)$, and $\mathscr{T}_{\mathscr{H}}(p)=\mathscr{H}_{j} \mathscr{T}(p)=\mathscr{H}_{j}(p)$, which imply that $\forall i, j, \mathscr{H}_{j}(p)$, and $\operatorname{Int}_{i}(p)$ are other fixed points of mapping $\{\mathscr{T}, \mathscr{H}\}$. For the uniqueness of $\mathscr{T}$ and $\mathscr{H}$ of self-mappings, we get $p=\mathscr{H}_{j}(p)=\mathscr{T}_{i}(p)$, which shows that $p$ is a common fixed point of $\mathscr{T}_{j}$ and $\mathscr{H}_{i}, \forall i, j$.

Example 5. Let $(\Gamma, \psi, \phi, *, \diamond)$ be a complete neutrosophic cone metric space, $k=\mathbb{R}^{+}$, and $\Gamma=[0, \infty)$. Define $\Phi=\Psi:[0, \infty) \longrightarrow[0, \infty)$ by $\Phi(m)=(m / 2), \Psi(m)=$ $(m / 4)$, for all $m \gg \phi$ and two families of self mappings $\mathscr{T}_{j}$ and $\mathscr{H}_{i}$ where $i, j \in\{1,2, \ldots, n\}$ by

$$
\begin{aligned}
& \mathscr{T}_{j}(x)= \begin{cases}0, & \text { if } m=0_{\phi}, \\
\frac{1}{x \sqrt{[n]} 6}, & \text { if } m>0_{\phi},\end{cases} \\
& \mathscr{H}_{i}(x)= \begin{cases}0, & \text { if } m>0_{\phi}, \\
\frac{1}{x^{\sqrt{[n]} 2}}, & \text { if } m=0_{\phi} .\end{cases}
\end{aligned}
$$

Then, we have

$$
\begin{aligned}
\Phi( & \left.\frac{1}{\psi(\mathscr{T}(t), \mathscr{T}(s), \mathscr{T}(r), m)}-1_{\phi}\right) \\
& -\Psi\left(\frac{1}{\psi(\mathscr{T}(t), \mathscr{T}(s), \mathscr{T}(r), m)}-1_{\phi}\right) \\
\quad= & \frac{3\left(z^{6}\left|t^{6}+s^{6}\right|+t^{6}\left|s^{6}+r^{6}\right|+s^{6}\left|r^{6}+t^{6}\right|\right)}{2 m t^{6} s^{6} r^{6}} \\
\quad \geq & \frac{z^{2}\left|t^{2}+s^{2}\right|+t^{2}\left|s^{2}+r^{2}\right|+s^{2}\left|r^{2}+t^{2}\right|}{2 m t^{2} s^{2} r^{2}} \\
\quad= & 1\left(\frac{1}{\psi(\mathscr{H}(t), \mathscr{H}(s), \mathscr{H}(r), m)}-1_{\phi}\right) .
\end{aligned}
$$

From the above and the idea of $\phi=1-\psi$, we get that statements (i) and (ii) hold. All statements of Theorem 2 hold; therefore, $\mathscr{T}_{j}$ and $\mathscr{H}_{i}$ have uniqueness.

\section{Conclusion}

In this paper, the definition of the neutrosophic cone metric space is introduced and studied. Based on this definition, we also stated and proved some fixed point theorems on the neutrosophic CMS. We provided a description of the example and investigated some properties in Section 3. We established and extended the definition of the $(\Phi, \Psi)$-weak contraction in the intuitionistic generalized fuzzy cone metric space.

\section{Data Availability}

No data were used to support the findings of this study.

\section{Conflicts of Interest}

The authors declare that there are no conflicts of interest regarding this manuscript.

\section{References}

[1] L. A. Zadeh, "Fuzzy sets," Information and Control, vol. 8, no. 3, pp. 338-353, 1965.

[2] C. L. Chang, "Fuzzy topological spaces," Journal of Mathematical Analysis and Applications, vol. 24, no. 1, pp. 182-190, 1968.

[3] K. Atanassov, "Intuitionistic fuzzy sets," Fuzzy Sets and Systems, vol. 20, no. 1, pp. 87-96, 1984.

[4] K. Atanassov and A. K. Stoeva, "Intuitionistic l-fuzzy," in Cybernetic and System Research, R. Trpple, Ed., vol. 2, pp. 539-540, Elsevier, Amsterdam, Netherlands, 1984.

[5] D. Çoker, "An introduction to intuitionistic fuzzy topological spaces," Fuzzy Sets and Systems, vol. 88, no. 1, pp. 81-89, 1997.

[6] G. Balasubramanian and P. Sundaram, "On some generalizations of fuzzy continuous functions," Fuzzy Sets and Systems, vol. 86, no. 1, pp. 93-100, 1997.

[7] F. Smarandache, "Neutrosophic set is a generalization of intuitionistic fuzzy set, inconsistent intuitionistic fuzzy set (picture fuzzy set, ternary fuzzy set), pythagorean fuzzy set (atanassov's intuitionistic fuzzy set of second type), q-rung orthopair fuzzy set, spherical fuzzy set, and $n$-hyperspherical fuzzy set, while neutrosophication is a generalization of regret theory, grey system theory, and three-ways decision, arxiv preprint arxiv: 1911.07333," Journal of New Theory, vol. 29, pp. 01-35, 2019.

[8] F. Smarandache, Neutrosophic Probability, Set, and Logic, ProQuest Information, Learning, Ann Arbor, MI, USA, 1998.

[9] M. A. A. Shumrani and F. Smarandache, "Introduction to non-standard neutrosophic topology," Symmetry, vol. 11, pp. 1-14, 2019.

[10] W. F. Al-Omeri and F. Smarandache, New Neutrosophic Sets via Neutrosophic Topological Spaces, Pons Publishing House, Brussels, Belgium, 2017.

[11] W. F. Al-Omeri, "Neutrosophic crisp sets via neutrosophic crisp topological spaces," Neutrosophic Sets and Systems, vol. 13, pp. 96-104, 2016.

[12] W. F. Al-Omeri, S. Jafari, and F. Smarandache, "Neutrosophic fixed point theorems and cone metric spaces," Neutrosophic Sets and Systems, vol. 31, no. 1, p. 18, 2020.

[13] A. George and P. Veeramani, "On some results in fuzzy metric spaces,” Fuzzy Sets and Systems, vol. 64, no. 3, pp. 395-399, 1994.

[14] F. Smarandache, "Neutroalgebra is a generalization of partial algebra," International Journal of Neutrosophic Science (IJNS), vol. 2, no. 1, pp. 08-17, 2020.

[15] F. Smarandache, "Introduction to neutroalgebraic structures and antialgebraic structures," in Advances of Standard and Nonstandard Neutrosophic Theories Ch. 6 (20220190), pp. 240-265, Pons Publishing House, Brussels, Belgium, 2020.

[16] L.-G. Huang and X. Zhang, "Cone metric spaces and fixed point theorems of contractive mappings," Journal of Mathematical Analysis and Applications, vol. 332, no. 2, pp. 1468-1476, 2007.

[17] T. Öner, M. B. Kandemir, and B. Tanay, "Fuzzy cone metric spaces," Journal of Nonlinear Sciences and Applications, vol. 8, no. 5, pp. 610-616, 2015.

[18] I. Beg, C. Vetro, D. Gopal, and M. Imdad, “ $(\phi, \psi)$-weak contractions in intuitionistic fuzzy metric spaces," Journal of 
Intelligent \& Fuzzy Systems, vol. 26, no. 5, pp. 2497-2504, 2014.

[19] V. Gupta, R. Saini, and A. Kanwar, "Some coupled fixed point results on modified intuitionistic fuzzy metric spaces and application to integral type contraction," Iranian Journal of Fuzzy Systems, vol. 14, no. 5, pp. 123-137, 2017.

[20] S. Wasfi, G. Vishal, and K. Ashima, "New results on modified intuitionistic generalized fuzzy metric spaces by employing e.a property and common e.a property for coupled maps," Journal of Intelligent and Fuzzy Systems, vol. 38, no. 3, pp. 3003-3010, 2020.

[21] A. A. Salama, S. Broumi, S. Broumi, and S. A. Alblow, "Introduction to neutrosophic topological spatial region, possible application to gis topological rules," International Journal of Information Engineering and Electronic Business, vol. 6, no. 6, pp. 15-21, 2014.

[22] A. A. Salama, F. Smarandache, and V. Kroumov, "Neutrosophic closed set and neutrosophic continuous functions," Neutrosophic Sets and Systems, vol. 4, pp. 4-8, 2014.

[23] W. Al-Omeri and S. Jafari, "On generalized closed sets and generalized pre-closed sets in neutrosophic topological spaces," Mathematics, vol. 7, no. 1, pp. 1-12, 2019. 